\title{
ORTHOGONAL TIME-VARYING FILTER BANKS AND WAVELETS
}

\author{
Cormac Herley and Martin Vetterli * \\ Department of Electrical Engineering \\ and Center for Telecommunications Research \\ Columbia University, New York, NY 10027-6699
}

\begin{abstract}
We consider the construction of time-varying orthogonal filter banks. We first show that implementing an orthogonal finite impulse response filter bank over a finite signal segment involves finding a set of orthogonal boundary filters. We show that by carrying out a Gram-Schmidt orthogonalization procedure we generate boundary filters, that necessarily remain localized in the region of the boundary. We give a complete constructive characterization of such boundaries for two-channel finite impulse response filter banks. These boundary constructions allow us to change the topology of orthogonal subband trees at will, by growing or pruning branches at any time. The boundary filter case can be further generalized to give overlapping transition filters when changing between orthogonal structures. If we use the time-varying filter banks in an iterated scheme they converge to continuous-time bases, much as in the non-time-varying case.
\end{abstract}

\section{INTRODUCTION}

Two-channel multirate filter banks, such as indicated in Figure 1, have been actively studied for some time. This structure has been widely used for subband coding, and the conditions under which it achieves perfect reconstruction are thoroughly understood. A very popular solution is that where perfect reconstruction is achieved with $H_{1}(z)=z^{N-1} H_{0}\left(-z^{-1}\right), N$ being the filter length of $H_{0}(z)$, and $G_{0}(z)=H_{0}\left(z^{-1}\right), G_{1}(z)=H_{1}\left(z^{-1}\right)$. This is known as the orthogonal solution, since it can be easily verified that

$$
<h_{1}(n), h_{0}(n-2 k)>=0
$$

$\left.<h_{0}(n), h_{0}(n-2 k)\right\rangle=\delta_{k}=\left\langle h_{1}(n), h_{1}(n-2 k)\right\rangle$,

i.e. the filter impulse responses are orthogonal with respect to even shifts.

The action of the filter bank on an infinite signal column vector $x$ can be represented using the time domain operators $\mathbf{H}_{0}$ and $\mathbf{H}_{1}$, where the $k$-th row of $\mathbf{H}_{0}$ is $h_{0}(n-2 k)$, and the $k$-th row of $\mathbf{H}_{1}$ is $h_{1}(n-$ $2 k)=(-1)^{n-1} h_{0}\left(N_{0}-n+2 k\right)[9,7]$. The two relations (1), and (2), together with the fact that Figure 1 is a perfect reconstruction system, implies that if we interleave the rows of $\mathrm{H}_{0}$ and $\mathrm{H}_{1}$ to form the matrix $\mathbf{T}$

"Work suppurted in part by the National Science Foundation under grants ECD-88-11111 and MIP-90-14189.

0-7803-1254-6/93\$03.00 @ 1993 IEEE

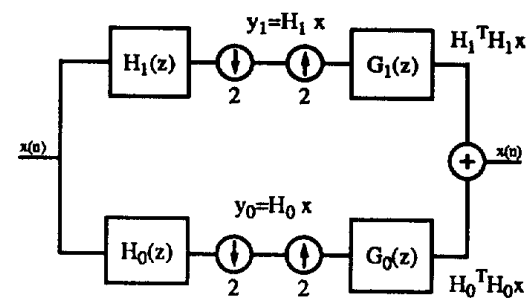

Figure 1: Maximally decimated two-channel multirate filter bank.

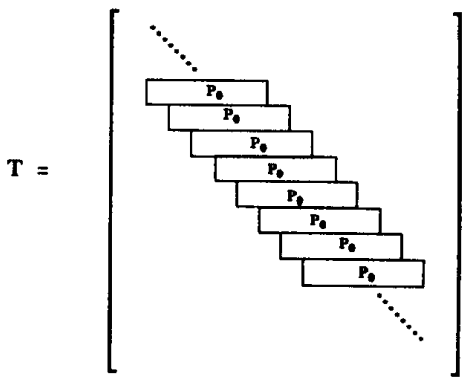

where $\mathbf{P}_{0}$ is the block

$$
\mathbf{P}_{0}=\left[\begin{array}{cccc}
h_{0}(0) & h_{0}(1) & \cdots & h_{0}\left(N_{0}-1\right) \\
-h_{0}\left(N_{0}-1\right) & h_{0}\left(N_{0}-2\right) & \cdots & h_{0}(0)
\end{array}\right]
$$

we get that

$$
\mathbf{T T}^{*}=\mathbf{T}^{*} \mathbf{T}=\mathbf{I} .
$$

In words, $\mathbf{T}$ is a doubly infinite unitary matrix, or the rows of $\mathrm{T}$ form an orthonormal basis for $l^{2}(Z)$, the space of square summable sequences.

A constraint of the analysis using this scheme is that it is necessarily stationary; that is it assumes that we are dealing with infinite signals, and that we use the same set of filters for all time. It is always possible for the finite duration signal problem to zero-pad the signal, but this means that there is some redundancy, and hence orthogonality is lost. Similarly one can switch filters at any time, but the perfect reconstruction property will be lost (at least in the transition).

The contribution of this paper is to show that it is possible to apply orthogonal filter banks to finite duration signals, and to change the filters used in the analysis (or indeed 
the topology of a filter bank tree) without losing orthogo nality or perfect reconstruction. Solutions to the finite duration signal problem have been given before, notably using the symmetric extension method [8], but we are interested in the orthogonal case. Time-varying filter banks were considered in [6] using a different approach.

Applications of the time-varying filter solutions to the construction of arbitrary orthogonal tilings of the timefrequency plane are described in $[3,4]$. We will restrict our attention to the case of two-channel orthogonal FIR filter banks; greater detail on many of the topics, and extension to the $M$-channel case are given in [5]. In the case of stationary two-channel filter banks there is a well-documented link with bases of wavelets [2]; we show that iteration of discretetime time-varying bases can lead to continuous-time timevarying bases in much the same way.

\section{BOUNDARY FILTERS}

Implementing an FIR filter bank over a finite signal segment involves finding some special way of treating the boundaries. Since we want the resulting operator to have the properties of orthogonality and perfect reconstruction (just as in the infinite duration case) we require a square unitary matrix. If we take, for example, the case of length-4 filters, applied for the segment $0 \leq n \leq n_{1}$, consider the following truncation of the time-domain operator

$$
\mathbf{M}=\left[\begin{array}{cccccc}
h_{0}(1) & h_{0}(2) & h_{0}(3) & 0 & 0 & \cdots \\
h_{0}(2) & -h_{0}(1) & h_{0}(0) & 0 & 0 & \cdots \\
0 & h_{0}(0) & h_{0}(1) & h_{0}(2) & h_{0}(3) & \cdots \\
0 & -h_{0}(3) & h_{0}(2) & -h_{0}(1) & h_{0}(0) & \cdots \\
\vdots & \vdots & \ddots & \ddots & \ddots & \ddots
\end{array}\right]
$$

We have shown the top left corner only, but the bottom right is entirely similar. It is easy to verify that this matrix is square, has full rank, but is no longer unitary. If we denote by $M_{i}$ the $i$-th row of $\mathrm{M}$ we find that

$$
<M_{i}, M_{j}>=0, \quad i \in\{0,1\}, j \in\left\{2,3, \cdots n_{1}-1, n_{1}\right\}
$$

but $\left\langle M_{0}, M_{1}\right\rangle \neq 0$ and $\left\langle M_{n_{1}-1}, M_{n_{1}}\right\rangle \neq 0$. Since the matrix is of full rank, i.e. we have a set of linearly independent vectors, we can restore orthogonality using the Gram-Schmidt procedure. To do this start by normalizing the first vector $M_{0}^{\prime \prime}=M_{0} /\left\|M_{0}\right\|$, and then

$$
\begin{aligned}
M_{1}^{\prime} & =M_{1}-<M_{1}, M_{0}^{\prime \prime}>M_{0}^{\prime \prime}-\sum_{j=3}^{n_{1}}<M_{1}, M_{j}>M_{j} \\
& =M_{1}-<M_{1}, M_{0}^{\prime \prime}>M_{0}^{\prime \prime} .
\end{aligned}
$$

The simplification is a consequence of (4). Finally set $M_{1}^{\prime \prime}=$ $M_{1}^{\prime} /\left\|M_{1}^{\prime}\right\|$. Note that since $M_{1}$ and $M_{0}^{\prime \prime}$ each have only three non-zero entries, so does $M_{1}^{\prime \prime}$ from (5). The same procedure is applied to the other boundary vectors $M_{n_{1-1}}$ and $M_{n_{1}}$. A new matrix $M^{\prime \prime}$ which has rows

$$
\left\{M_{0}^{\prime \prime}, M_{1}^{\prime \prime}, M_{2}, M_{3}, \cdots, M_{n_{1}-2}, M_{n_{1}-1}^{\prime \prime}, M_{n_{1}}^{\prime \prime}\right\}
$$

is then obviously unitary. What is important to note is that $M^{\prime \prime}$ has exactly the same zero entries as $M$; i.e. the boundary filters have the same support as the truncated filters.

One might expect that this example merely illustrates a peculiarity of the length-4 case; but this is not so. For any two-channel FIR filter bank the boundary vectors always have non-zero elements only in the region of the boundary.
Hence any possible completion of a truncated time domain matrix can be found just by using the Gram-Schmidt procedure. Formally we state this as follows.

Proposition 2.1 The set of boundary filters needed to apply a two-channel orthogonal filter bank, with length- $N$ filters to a finite length signal is a set of $(N-2) / 2+d$ vectors at each boundary, each of which has only $N-2+d$ non-zero values.

A proof is given in [5], we will here give the major steps. First consider a matrix $\mathbf{G}$ which contains a truncated version of the infinite time domain matrix, but where (in contrast to (3)) none of the filters are "chopped off." That is

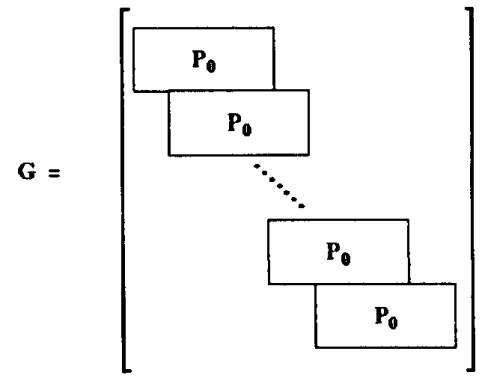

where $\mathbf{P}_{0}$ is the block of impulse response coefficients already defined. The dimension of $\mathrm{G}$ is clearly $2 k \times N_{0}+$ $2(k-1)$, where $k$ is the number of blocks. Next form the matrix

$$
\mathbf{Q}=\left[\begin{array}{lll}
\mathbf{O}_{d_{\mathbf{l}}} & \mathbf{G} & \mathbf{O}_{d_{r}}
\end{array}\right],
$$

where $\mathbf{O}_{d_{1}}$ and $\mathbf{O}_{d_{r}}$ are $2 k \times d_{l}$ and $2 k \times d_{r}$ zeros matrices. We shall use $\mathbf{Q}$ to construct an orthogonal filter bank for a signal of length $L=N_{0}+2(k-1)+d_{l}+d_{r}$. We already have $2 k$ basis vectors (the rows of $\mathbf{Q}$ ) so we must find the $L-2 k=N_{0}-2+d_{l}+d_{r}$ remaining vectors necessary to complete the basis.

Now note, from standard linear algebra, that $\mathbf{P}=(\mathbf{I}-$ $\mathbf{Q}^{T} \mathbf{Q}$ ) is the orthogonal projection onto the space orthogonal to the row space of $\mathbf{Q}$. Hence, any row suitable to be added to the list of basis vectors must have the form

$$
\mathbf{e}_{i}^{\prime}=\left(\mathbf{I}-\mathbf{Q}^{T} \mathbf{Q}\right) \cdot \mathbf{e}_{i},
$$

for some $e_{i}$. In fact the Gram-Schmidt procedure is to choose $L-2 k$ vectors linearly independent of the existing $2 k$ rows of $\mathbf{Q}$ and of each other; orthogonalize the first $\mathbf{e}_{0}$ with respect to the others by taking $\mathbf{e}_{0}^{\prime}=\mathbf{P} \cdot \mathbf{e}_{0}$, and form a new matrix $\mathbf{Q}_{1}$ by adding the normalized vector $\mathbf{e}_{0}^{\prime \prime}=\mathbf{e}_{0}^{\prime} /\left\|\mathbf{e}_{0}^{\prime}\right\|$. The second vector is orthogonalized using the projection $\mathbf{P}=\left(\mathbf{I}-\mathbf{Q}_{1}^{T} \mathbf{Q}_{1}\right)$ and so on.

The critical observation, proved in [5], is that $\left(I-Q^{T} Q\right)$ has the following form

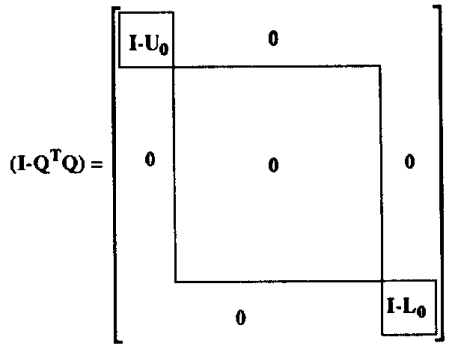


where the blocks $U_{0}$ and $L_{0}$ are square matrices of size $\left(N_{0}-2\right) / 2+d_{l}$ and $\left(N_{0}-2\right) / 2+d_{r}$ respectively. Hence the projection matrix is zero apart from the small blocks in the upper left and lower right corners. Thus all of the orthogonalized vectors $e_{i}^{\prime \prime}$ will have non-zero entries only in the first and/or last $N-1$ positions. When the signal length $L$ is large enough (roughly twice the filter length) the two non-zero portions of the Gram-Schmidt outputs $\mathbf{e}_{i}^{\prime}$ are non-overlapping. In this case we can choose half of the inputs to the procedure to have zeros in the last $N-1$ positions, the corresponding outputs will then have no-zero values only in the first $N-1$ positions. Similarly the second half of the Gram-Schmidt inputs can be chosen such that the outputs have non-zero values only in the last $N-1$ positions. The $N=4$ case constructed first was of this form, so that there were two filters each with three nonzero values at each boundary. Solutions which have nonzero values at both boundaries correspond to the circulant solutions and variations.

Essentially, once given enough linearly independent vectors to complete the basis, the Gram-Schmidt procedure just orthogonalizes them. If we change the input vectors, then we get a different orthogonal set at the output. Obviously the procedure is not unique. Given one solution, we can however search the space of all possible solutions by premultiplying by the matrix

$$
\left[\begin{array}{ccc}
\mathrm{U}_{l} & \mathbf{0} & \mathbf{0} \\
\mathbf{0} & \mathbf{I}_{n_{1}-4} & \mathbf{0} \\
\mathbf{0} & \mathbf{0} & \mathrm{U}_{r}
\end{array}\right],
$$

where $U_{l}$ and $U_{r}$ are unitary matrices of size $\left(N_{0}-2\right) / 2+d_{l}$ and $\left(N_{0}-2\right) / 2+d_{r}$ respectiveley. Minimal parameterizations of such matrices are easily realized in terms of rotation elements.

\section{TRANSITIONS BETWEEN FILTER BANKS}

We can use the boundary filters generated above to switch between orthogonal filter banks. This is easily done since if $M_{a}$ and $M_{b}$ are the matrices representing two different filter banks operating over finite signal segments then

$$
\left[\begin{array}{cc}
\mathbf{M}_{a} & \mathbf{0} \\
\mathbf{0} & \mathbf{M}_{b}
\end{array}\right]
$$

clearly implements a boundary between the filter banks. The above matrix is obviously unitary. The matrices $\mathbf{M}_{a}$ and $\mathbf{M}_{b}$ need not represent finite duration filter banks; if $\mathbf{M}_{a}$ were half-infinite with boundary filters in the lower right corner, and $\mathrm{M}_{b}$ half-infinite with boundary filters in the upper left we should still get a unitary matrix. This would give us a decomposition of an infinite signal, but where we change filters at some point in the processing.

For this transition obviously there is no overlap between the boundary filters of the $\mathbf{M}_{a}$ and $\mathbf{M}_{b}$ blocks; orthogonality is trivially obvious. It is also possible to have transition filters such that they have support on both sides of the boundary. We can explore the whole set of transition filters by premultiplying by a matrix

$$
\left[\begin{array}{ccc}
\mathbf{I} & \mathbf{0} & \mathbf{0} \\
\mathbf{0} & \mathbf{U}_{d} & \mathbf{0} \\
\mathbf{0} & \mathbf{0} & \mathbf{I}
\end{array}\right]
$$

where $d$ is the number of vectors in the transition block, i.e. the total number of boundary vectors on both sides of the transition. In general this produces overlapping transition functions, except in the particular case where $\mathbf{U}_{d}$ is block diagonal and preserves the boundary filter pattern. (a)

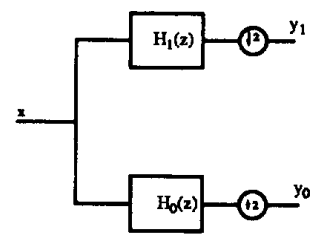

(b)

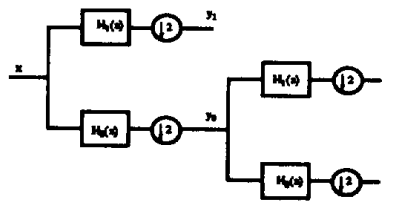

Figure 2: Splitting of the input signal $\mathbf{x}$ using orthogonal two channel filter banks. (a) Single division. (b) Iterated division.

Growing a subband tree, for example in changing from the structure of Figure 2 (a) to that of Figure 2 (b) or the reverse. This would make it possible to improve the frequency resolution (going from (a) to (b)) or the time resolution (from (b) to (a)) in a time-varying fashion.

\section{CONTINUOUS-TIME BASES}

In [2] it was demonstrated how discrete-time bases derived from two-channel filter banks could be iterated to derive continuous-time bases of wavelets. It turns out that a similar results holds in the time-varying case: we can use the discrete-time methods that we have derived to generate continuous-time bases.

We will here sketch the idea, leaving a detailed analysis to [5]. We consider the boundary filter case only, and, for simplicity, examine the example where there is only one boundary. For example suppose the matrix $\mathbf{M}_{b}$ in (7) to be half infinite, and to have boundary filters in the upper left corner; its rows form an orthonormal basis for half-infinite right-sided sequences. Call the matrix which contains its even-indexed rows $\mathbf{H}_{0}$, and the matrix with its odd-indexed rows $H_{1}$. We will iterate this filter bank by considering the matrix products $\mathbf{H}_{0}^{k}$.

Denote by $L_{i k}(z)$ the $z$-transform of the coefficients of the i-th row of $\left(\mathrm{H}_{0}\right)^{k}$ :

$$
L_{i k}(z)=\sum_{n=-\infty}^{\infty} \mathbf{H}_{0}^{k}(i, n) z^{-n} .
$$

It can now be shown that, for some small $i_{0}$ (less than twice the number of boundary filters) that

$$
L_{i k}(z)=z^{-1-2^{k}\left(i-i_{0}\right)} \prod_{p=0}^{k-1} H_{0}\left(z^{2^{p}}\right) \quad i>i_{0} .
$$

The function $L_{i_{0}, k}(z)$ can easily be recognized as the $z-$ transform of the "graphical iteration" [1] to find the scaling function $\phi_{h}(x)$ corresponding to the filter $H_{0}(z)$ (see [10]). That is if we define from $L_{i k}(z)$ a continuous-time function

$$
f_{i}^{(k)}(x)=\mathbf{H}_{0}^{k}(i, j) \quad j / 2^{k} \leq x<(j+1) / 2^{k},
$$




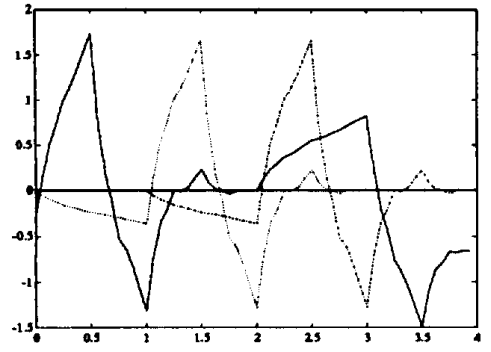

Figure 3: Boundary functions for wavelet basis for the interval $[0,4)$. Left and right boundary functions at one scale are shown; these are supported on $[0,2)$ and $[2,4)$ respectively. The wavelets $\psi_{h}(x), \psi_{h}(x-1)$ are also shown.

it can be shown that for $i \geq i_{0} f_{i}^{(k)}(x)$ converges to the scaling function $\phi_{h}\left(x+i_{0}-i\right)=\phi_{1}(x)$ as $k \rightarrow \infty$.

Thus the rows $i \geq i_{0}$ converge to $\phi_{h}\left(x+i_{0}-i\right)$. But what of the rows $i<i_{0}$ ? This question is answered by the next proposition.

Proposition 4.1 The sequences $f^{(k)}(x)$ for $0 \leq i<i_{0}$ converge for $x>\epsilon$, provided that for and $i=i_{0}$ does. Further, given $\epsilon>0$ when $0 \leq i<i_{0}$ we find that $\phi_{i}(x)$ is a finite linear combination of $\phi_{h}(x-m)$ for $x>\epsilon$.

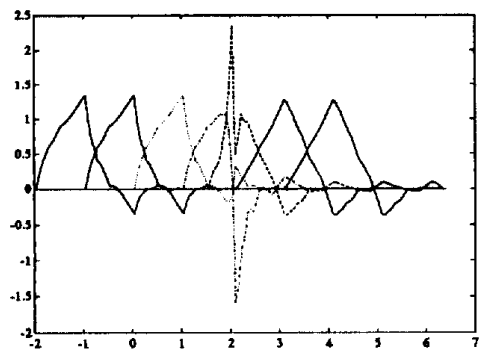

Figure 4: Transition functions for the transition between $\phi_{2}(x)$ and $\phi_{3}(x)$. There are three orthogonal transition functions shown, which span the null space between $\left\{\phi_{2}(x+\right.$ $\left.1), \phi_{2}(x+2), \cdots\right\}$ and $\left\{\phi_{3}(x-2), \phi_{3}(x-3), \cdots\right\}$.

We call the $\phi_{i}(x)$ for $i<i_{0}$ the boundary scaling functions. Again, following the analogy with the non-timevarying case, to complement the scaling functions we define

$$
s_{i}^{(k)}(x)=\left(\mathbf{H}_{1} \cdot \mathbf{H}_{0}^{k}\right)(i, j) \quad j / 2^{k} \leq x<(j+1) / 2^{k} .
$$

Under the same conditions the $s_{i}^{(k)}(x)$ converge to $\psi_{h}(x+$ $\left.i_{0}-i\right)$ for $i \geq i_{0}$. For $0 \leq i<i_{0}$ the $s_{i}^{(k)}(x)$ converge to functions $\psi_{0}(x), \psi_{1}(x), \cdots \bar{\psi}_{i_{0}}(x)$, which we call the transition wavelets. It is a direct consequence of Proposition 4.1 that these sequences converge for $x>\epsilon$ and have the same smoothness as $\psi_{h}(x)$ away from the boundary.

Because of the orthogonality of all rows of $\mathbf{M}_{b}$ we have

$$
\mathbf{H}_{\mathbf{0}} \cdot \mathbf{H}_{\mathbf{0}}^{*}=\mathbf{I} \text { and } \mathbf{H}_{\mathbf{0}} \cdot \mathbf{H}_{\mathbf{1}}^{*}=\mathbf{0} \text {. }
$$

This guarantees

$$
<\phi_{n}(x), \psi_{p}(x)>=\delta_{n p} .
$$

So the transiton scaling functions and wavelets are orthogonal as expected. To show orthogonality of the wavelets across scale note that the wavelet at a scale $k$ is found from the rows of $\mathbf{H}_{1} \cdot \mathbf{H}_{0}^{\mathbf{k}}$. So to show orthogonality of the wavelets at scales $k$ and $k-1$ we observe:

$$
\begin{aligned}
\mathrm{H}_{1} \cdot \mathbf{H}_{0}^{\mathbf{k}} \cdot\left(\mathrm{H}_{0}^{*}\right)^{\mathbf{k}-1} \cdot \mathrm{H}_{1}^{*} & =\mathrm{H}_{1} \cdot \mathrm{H}_{0} \cdot \mathbf{H}_{1}^{*} \\
& =0 .
\end{aligned}
$$

Thus

$$
\left\langle\psi_{n}(x), \psi_{p}(2 x)\right\rangle=0
$$

This is the machinery we need to derive wavelet bases for the interval.

An example is shown in Figure 3, where the left and right boundary wavelet functions are shown, along with $\psi_{h}(x)$ and $\psi_{h}(x-1)$. The Daubechies length-4 filters were used in generating this example.

We can also take iterates of a discrete-time basis containing transition filters. An example of the iteration of such a scheme, using the same filters, is given in Figure 4.

\section{REFERENCES}

[1] I. Daubechies. Orthonormal bases of compactly supported wavelets. Communications on Pure and Applied Mathematics, XLI:909-996, 1988.

[2] I. Daubechies. Ten Lectures on Wavelets. SIAM, 1992.

[3] C. Herley, J. Kovačević, K. Ramchandran, and M. Vetterli. Arbitrary orthogonal tilings of the time-frequency plane. IEEE Trans. Signal Proc., Special Issue on Wavelets, 1992. Submitted.

4] C. Herley, J. Kovacević, K. Ramchandran, and M. Vetterli. Time-varying orthonormal tilings of the timefrequency plane. In Proc. IEEE Int. Conf. ASSP, Minneapolis, MN, April 1993. To appear.

[5] C. Herley and M. Vetterli. Orthogonal time-varying filter banks and wavelets. IEEE Trans. on Signal Proc. 1993. Submitted.

[6] K. Nayebi, T. P. Barnwell III, and M. J. T. Smith. Analysis-synthesis systems with time-varying filter bank structures. In Proc. IEEE Int. Conf. ASSP, pages 617-620, San Francisco, CA, March 1992

[7] K. Nayebi, T. P. Barnwell III, and M. J. T. Smith. Time domain filter bank analysis: A new design theory. IEEE Trans. on Signal Proc., IV(6):1414-1429, June 1992 .

[8] M. J. T. Smith and S. L. Eddins. Analysis/Synthesis techniques for subband image coding. IEEE Trans. Acoust., Speech, Signal Proc., 38(8):1446-1456, August 1990.

[9] M. Vetterli and D. Le Gall. Perfect reconstruction FIR filter banks: some properties and factorizations. IEEE Trans. Acoust., Speech, Signal Proc., 37(7):1057-1071, July 1989 .

[10] M. Vetterli and C. Herley. Wavelets and filter banks: theory and design. IEEE Trans. on Signal Proc., 40(9):2207-2232, Sept. 1992. 